\title{
Characterization of Nanoplastics, Fibrils, and Microplastics Released during Washing and Abrasion of Polyester Textiles
}

Tong Yang, Jialuo Luo, and Bernd Nowack*

Cite This: Environ. Sci. Technol. 2021, 55, 15873-15881

Read Online

ABSTRACT: Nanoplastics (defined here as plastic particles smaller than $1000 \mathrm{~nm}$ ) released during the daily use of plastic products are gaining increasing attention due to their potential effects on human and environmental health. Formation of nanoplastics has been reported so far for diverse plastic products under varying conditions of use. The washing of synthetic textiles has been identified as an important source of microplastic fibers

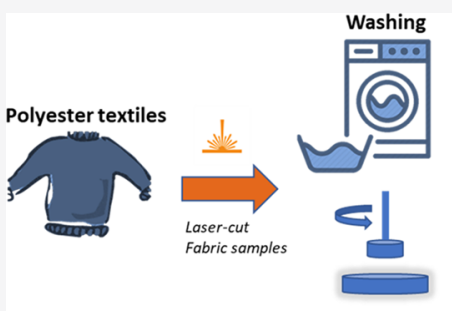

Abrasion

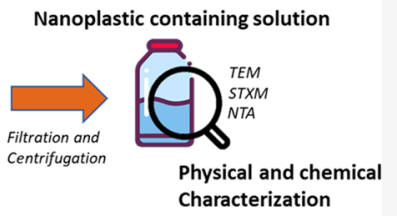
textiles was shown to induce further fragmentation of fibers and subsequent formation of much smaller and shorter fibrils. The aim of this work was to identify whether washing and wearing of textiles also results in the formation of nanoplastics. We designed washing and abrasion experiments to investigate the morphology, number, and size of micro- and nanoplastics released from polyester textiles. Using a combination of techniques including scanning transmission X-ray microspectroscopy (STXM), scanning electron microscopy (SEM), transmission electron microscopy (TEM), and nanoparticle tracking analysis (NTA), we were able to quantify nanoplastics (average hydrodynamic diameter 173-188 nm), microplastic fibrils (diameter $3 \pm 1 \mu \mathrm{m}$, length $20-160 \mu \mathrm{m}$ ), and MPFs (diameter $16 \pm 7 \mu \mathrm{m}$, length up to $5 \mathrm{~mm}$ ). The presence of polyester nanoplastics was confirmed by the near edge X-ray absorption fine spectra (NEXAFS) of the nanoparticles in the abrasion and washing samples for particles larger than $100 \mathrm{~nm}$. We estimated that in the abraded samples, $1 \mathrm{~g}$ of fleece textile released an average of $2.1 \times 10^{11}$ nanoplastic particles $(1.4 \mathrm{mg}), 1.4 \times 10^{4}$ MPFs $(1.0 \mathrm{mg})$, and $5.3 \times 10^{5}$ fibrils $(0.5 \mathrm{mg})$ based on SEM images and NTA. In the nonabraded samples, $1 \mathrm{~g}$ of textile released an average of $3.3 \times 10^{11}$ nanoplastic particles $(2.1 \mathrm{mg}), 2.8 \times 10^{3} \mathrm{MPFs}(0.2 \mathrm{mg})$, and no fibrils. The present study is the first to show a significant release of polyester nanoplastics during the washing and abrasion of synthetic textiles.

KEYWORDS: polyester, nanoplastics, microplastic fibers, fibrils, washing, abrasion

\section{INTRODUCTION}

Microplastics, as one of the defining environmental pollutants of the Anthropocene, are receiving more and more attention. ${ }^{1}$ Microplastics are defined as plastic debris that is smaller than 5 mm. ${ }^{2,3}$ A recent mapping of global microplastic concentrations has shown multiple hotspots of microplastics in both sediments and surface waters worldwide. ${ }^{4}$ Fragmentation of plastic litter does not stop at the microplastic size, but smaller particles can also be formed, called nanoplastics, which are plastic particles smaller than $100 \mathrm{~nm}^{5,6}$ or smaller than 1000 $\mathrm{nm}$ in other definitions. ${ }^{7,8}$ In the present study, we define nanoplastics as plastic particles smaller than $1000 \mathrm{~nm}$. Microand nanoplastics are different with respect to many properties, including surface-related properties, fate and transport processes, biological interactions, and the different analytical techniques needed to characterize them. ${ }^{9,10}$

Several studies have investigated the possibility of plastic debris breaking down into nano-sized plastics by UV radiation, ${ }^{11}$ mechanical energy, ${ }^{12}$ and through digestive fragmentation. ${ }^{13}$ Furthermore, nanoplastics have been demonstrated to be released during the use of personal care products, ${ }^{6}$ the boiling of plastic teabags, ${ }^{14}$ and the use of milk bottles. ${ }^{15}$ Only a handful of studies confirmed so far the presence of nanoplastics in environmental samples. ${ }^{16}$ Wahl et al. ${ }^{17}$ concentrated and separated poly(vinylchloride) (PVC), polystyrene (PS), and polyethylene (PE) nanoplastics ranging from 20 to $150 \mathrm{~nm}$ from a soil sample. PVC, poly(ethylene terephthalate) (PET), PS, and PE nanoplastics were observed in the North Atlantic subtropical gyre. ${ }^{18}$ However, both studies did not quantify the concentrations of the identified nanoplastics. One study reported that the concentration of PET nanoplastics smaller than $200 \mathrm{~nm}$ in Alpine surface snow samples was 5-25 ng/mL. ${ }^{19}$ Furthermore, a recent study, which reviewed 33 studies on state-of-art methodologies for pretreatment, separation, identification, and quantification of

Received: July 19, 2021

Revised: October 26, 2021

Accepted: October 27, 2021

Published: November 16, 2021 
nanoplastics, has found that the major challenge of most studies was that the quantification was often conducted without chemical confirmation of polymer types, raising concerns about the reliability of current results. ${ }^{16}$

The possible adverse effects of nanoplastics include the inhibition of fish reproduction and of the growth of aquatic plants. $^{20,21}$ Besides, nanoplastics can also interact with plant roots and permeate our food chain. ${ }^{20}$ A recent meta-analysis of nanoplastic hazard reported safe concentrations for nanoplastics of $6.9 \times 10^{11}$ particles $/ \mathrm{m}^{3}$ in marine and $6.8 \times 10^{13}$ particles $/ \mathrm{m}^{3}$ in freshwater. ${ }^{22}$

Microplastic fibers (MPFs) have been identified as an important component of plastic pollution in water ${ }^{23,2423,24}$ and previous studies have suggested that domestic washing is a major pathway for MPF release. ${ }^{25}$ The MPFs released during washing of textiles exhibit a large variability of the number of released MPFs, ranging from a few to more than 10000 MPFs/g textile washed. ${ }^{26-33}$ A recently published paper identified that washing of abraded polyester textiles released not only MPFs (diameter 12-13 $\mu \mathrm{m}$, length $200-800 \mu \mathrm{m}$ ) but also fibrils with a much smaller size, ranging in length from 30 to $150 \mu \mathrm{m}$ and having a diameter between 2 and $5 \mu \mathrm{m}$. ${ }^{34}$ The number of fibrils extracted from the abraded samples was $6000 \pm 2700$ fibrils/g of textile, 3 times larger than the number of MPFs. ${ }^{34}$ The possible release of nanoplastics from textiles has so far not been investigated, but intuitively this could be an important part of the plastic profile in textile washing studies as fragmentation into smaller fiber fragments is clearly taking place.

Therefore, the objective of the present study was to comprehensively characterize nanoplastics and microplastics (MPF and fibrils) released during washing and abrasion of polyester textiles. Chemically, we used scanning transmission X-ray microspectroscopy (STXM) to obtain chemical information of the nanoplastics. For the physical information, nanoparticle tracking analysis (NTA) was used to report the size and number concentration of nanoparticles. Transmission electron microscopy (TEM) was also used as a supplementary method to capture the morphology and analyze the size distribution of the nanoparticles. Besides, we also characterized the number concentration and size distribution of the released MPFs and fibrils by scanning electron microscope (SEM) image analysis.

\section{MATERIALS AND METHODS}

A series of experiments were conducted, following the scheme depicted in Figure S1: Polyester fabrics were cut into samples by a laser cutter and then were abraded or not abraded before washing. The samples are referred to as "washing" or "abrasion" samples. The wash solutions were filtered, and the filters were characterized by SEM image analysis for MPF and fibrils. In the filtrate, nanoparticles were analyzed by NTA, TEM, and STXM for chemical and physical information. Triplicates of control samples without added textiles were prepared to quantify the background contamination with nanoparticles. The control samples went through the washing, filtration, and centrifugation processes and are referred to as "blank" samples. The detailed experimental information is documented in the following sections.

2.1. Textiles. Black-colored fleece polyester textiles with a density of $185 \pm 1 \mathrm{~g} / \mathrm{m}^{2}$ were used in the present study. The same textiles were also used and characterized in previous MPF studies, ${ }^{32,34}$ where more details can be found. Fleece was found to have one of the highest MPF releases among all of the types of fabrics tested. ${ }^{32,34}$ Therefore, we chose fleece to increase our chance of finding nanoplastics during washing and abrasion experiments. The fleece textiles were cut by a laser cutter (tt-1300, Times Technology) into $140 \mathrm{~mm}$ (abradant) and $38 \mathrm{~mm}$ (specimen) circular pieces. The average weight of the abradant was 2.83 and $0.21 \mathrm{~g}$ for the specimen. More details on the method can be found in the recent paper describing abrasion experiments with polyester fabrics. ${ }^{34}$

2.2. PET Reference Nanoparticles. PET nanoparticles were prepared following an established protocol. ${ }^{35}$ In the present study, $1 \mathrm{~g}$ of $300-\mu \mathrm{m}$-PET powder (Goodfellow Cambridge Limited) was dissolved in $10 \mathrm{~mL}$ aqueous trifluoroacetic acid (TFA) solution $(90 \% \mathrm{v} / \mathrm{v})$ at $50{ }^{\circ} \mathrm{C}$ under stirring (700 rpm) for $2 \mathrm{~h}$ and stored overnight. Briefly, $10 \mathrm{~mL}$ of aqueous TFA solution $(20 \% \mathrm{v} / \mathrm{v})$ was added dropwise to the resulting mixture under vigorous stirring and kept stirring overnight. The suspension was centrifuged at $2500 \mathrm{rpm}$ for $1 \mathrm{~h}$ to obtain a PET pellet. The pellet was then redispersed in $100 \mathrm{~mL}$ of $0.5 \%$ aqueous sodium dodecyl sulfate (SDS) solution. The obtained suspension was stirred for $1 \mathrm{~h}$ and ultrasonicated (SONOPULS HD 2070) for $5 \mathrm{~min}$ three times and poured into a cylinder. The top $20 \mathrm{~mL}$ of the PET suspension was taken after $1 \mathrm{~h}$ settlement and used for further characterization. The reference TEM grid was then prepared by drop-casting the suspension onto a TEM grid (EM Resolutions C200Cu25). TEM images of the PET reference nanoparticles can be found in Figure S2.

2.3. Abrasion. For the abrasion experiments, we used a Martindale tester (4-station Martindale tester SN-103/06/ 1049, James Heal) to rub pieces of fleece polyester fabrics against each other according to the procedure documented in ISO $129477-2: 2016 .{ }^{36}$ The Martindale method is widely used for testing the abrasion resistance of clothes and household textiles, decorative fabrics, and furniture. ${ }^{37}$ Before the abrasion test started, an air blower was used to reduce crosscontamination from the Martindale tester, and the apparatus was also cleaned by ethanol before abrasion. The abrasion test was performed with $12 \mathrm{~kg}$ pressure and was stopped after 5000 rubs, which is when the pilling phenomena can be observed. ${ }^{34}$ Pilling is commonly seen during the wearing of fleece sweaters. After the abrasion experiment, specimens (the $38 \mathrm{~mm}$ pieces) were removed and washed according to Section 2.4. The abrasion tests were performed in triplicate. Samples were prewashed once before the abrasion experiments.

2.4. Washing. A Gyrowash machine (James Heal, GyroWash Model 1615) with eight steel beakers was used to simulate a domestic washing process. The standard washing conditions were based on ISO $105-\mathrm{C}^{3} 6^{38}$ for colorfastness to domestic and commercial laundering with slight modifications. The washing conditions were the same as described in the previous microplastic washing paper. ${ }^{32}$ We used $100 \mathrm{~mL}$ of linear alkylbenzene sulfonic acid (LAS) solution $(0.75 \mathrm{~g} / \mathrm{L})$ as the washing solution. The $\mathrm{pH}$ value of the LAS solution was adjusted by $1 \mathrm{~mol} / \mathrm{L}$ sodium hydroxide solution to $9.2 \pm 0.1$. Each round of washing takes $45 \mathrm{~min}$ at a temperature of $40{ }^{\circ} \mathrm{C}$, and the speed of the rotating beakers was $40 \mathrm{rpm}$. All experiments were performed with three replicates. A $20 \mathrm{~min}$ prewash with LAS solution was performed before each washing to reduce dust and other contaminants. To minimize crosscontamination, glassware was applied instead of anything plastic. The textiles were prewashed once before the experiments. 


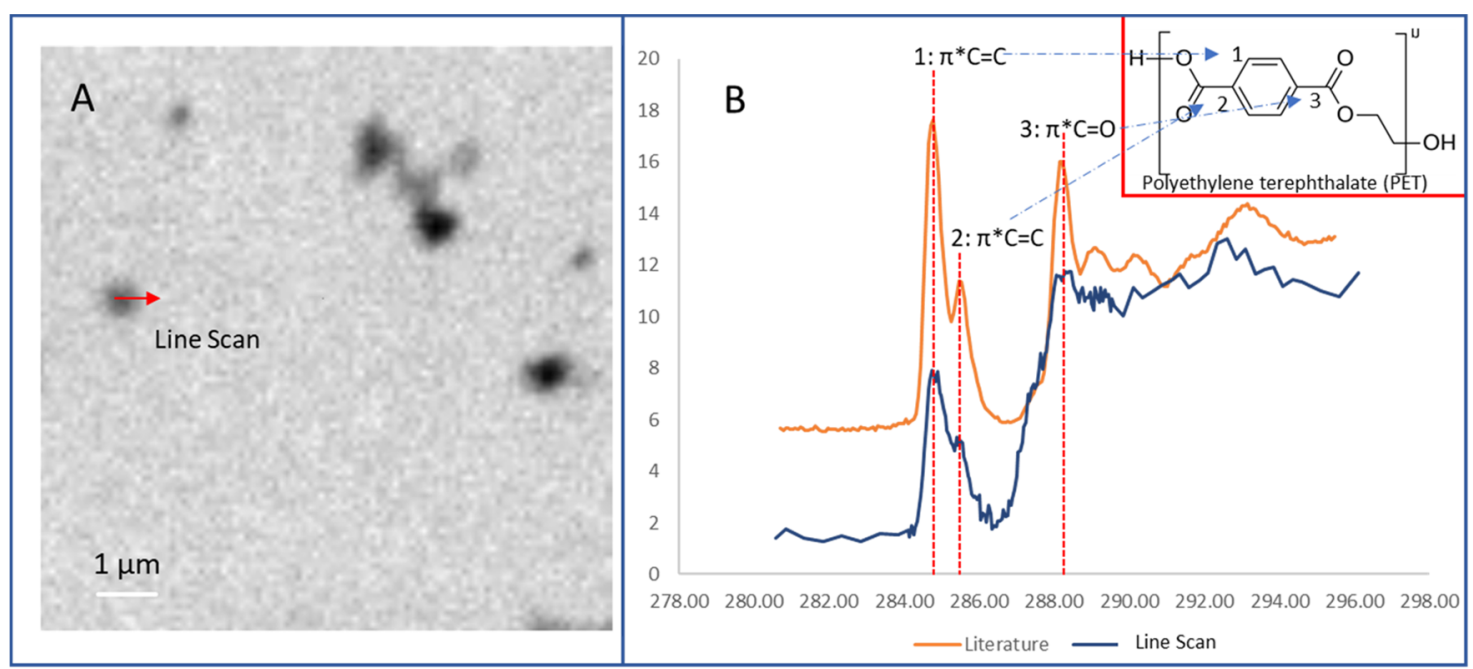

Figure 1. (A) STXM image of synthesized PET reference nanoparticles; (B) comparison of PET (thickness of about 100-150 nm) NEXAFS spectrum from the literature ${ }^{39}$ and the NEXAFS spectrum of the PET nanoparticle shown in (A). (1) $\pi^{*} \mathrm{C}=\mathrm{C}$ signal at $284.4 \mathrm{eV}$; $(2) \pi^{*} \mathrm{C}=\mathrm{C}$ signal at $285.1 \mathrm{eV}$; and $(3) \pi^{*} \mathrm{C}=\mathrm{O}$ at $287.8 \mathrm{eV}$.

2.5. Particle Separation and Isolation. After washing, the wet fabrics were squeezed, and the washing solutions were collected. About $10 \mathrm{~mL}$ solution was filtered through a $25 \mathrm{~mm}$ polycarbonate cyclopore membrane (Whatman Inc.) with a pore size of $2 \mu \mathrm{m}$. The filtrate was kept in glass bottles for nanoplastics characterization. A $25 \mathrm{~mm}$ cellulose acetate filter (Sartorius Stedim Biotech $\mathrm{GmbH}$ ) with a pore size of $0.45 \mu \mathrm{m}$ was used to collect MPF and fibrils on the filters.

The nanoparticles in the filtrate were then centrifuged onto a TEM grid (EM Resolution C200Cu25) at $3000 \mathrm{rpm}$ ( $G$ force $=754.6$ ) for $40 \mathrm{~min}$. A TEM grid was placed on a customized aluminum cone covered by a fresh piece of Parafilm, and then the cone was fitted into a $2 \mathrm{~mL}$ Eppendorf microcentrifuge tube. Then, $1 \mathrm{~mL}$ solution from each sample collected from the washing experiment was transferred into the microcentrifuge tube. After centrifugation, the grid was taken off the cone and used for STXM and TEM characterization. During this procedure, according to Stokes' Law, all PET particles that are larger than $200 \mathrm{~nm}$ are completely settled on the TEM grid. Detailed operations of the centrifugation were filmed and uploaded online, which can be found at the link provided in Figure S1.

2.6. Characterization. 2.6.1. STXM. We identified the chemical identity of the nanoparticles via near edge X-ray absorption fine spectroscopy (NEXAFS). The STXM images were taken at PSI Switzerland using the PolLux-X07DA beamline. STXM images at the carbon K-edge were recorded at $350 \mathrm{eV}$, and the corresponding NEXAFS spectra between 270 and $350 \mathrm{eV}$ were obtained with a dwell time of $40 \mathrm{~ms}$. A PET nanoparticle reference TEM grid was prepared by dropcasting, and particles of three size categories (100-200, 400500 , and $800-1000 \mathrm{~nm}$ ) were scanned for NEXAFS (4 spectra recorded). Besides the reference grid, three TEM grids from washing samples, three TEM grids from abrasion samples, and one grid from the method blank sample (with no textiles washed, only LAS solution) were prepared by centrifugation, as described in Section 2.5. NEXAFS spectra were processed in aXis2000 and Microsoft Excel with a default line fitting model which connects all data points.

2.6.2. TEM. Images were taken by JEOL JEM-1400 Plus at 5000-10000× magnification to obtain morphological in- formation of the particles. The size distribution of nanoparticles was determined by sampling and analysis of TEM images. For each TEM sample grid, inner/middle/outer rings are classified, as shown in Figure S3. Five grid openings are randomly sampled (one in the inner ring, two in the middle ring, and two in the outer ring). If the chosen grid opening was broken, it was replaced by an adjacent grid opening. For each grid opening, three images were taken at $4000 \times$ magnification.

2.6.3. NTA. Nanosight LM20 (Nanosight, England) was employed for NTA. A $500 \mu \mathrm{L}$ solution was introduced to the viewing cell, and $60 \mathrm{~s}$ videos were recorded for each sample. Particles were counted and analyzed by NTA image analysis software, giving the attenuated curves of particle size distribution and number concentration. After calibration with polystyrene nanoplastics (Thermo Scientific, Lot No.: 41118) with a size of $102 \pm 3 \mathrm{~nm}$, the linear working range was found to be between $10^{6}$ and $10^{9}$ particles/mL (Figure S4). We measured washing, abrasion, and method blank samples within this linear working range.

2.6.4. SEM. Filters were sputtered with a $7 \mathrm{~nm}$ layer of $\mathrm{Au} /$ Pd by a high vacuum sputter coater (Leica EM ACE600) before observation under SEM (7 kV, Quanta FEI 650). MPF and fibril number and length were determined using a SEM "mapping" method developed in a previous MPF study. ${ }^{34} \mathrm{~A}$ grid of $7 \times 10$ images was taken at $200 \times$ magnification with a width of $3.0673 \mathrm{~mm}$ and a resolution of $5582 \times 4601$ pixels. All MPF and fibrils on the filtered samples were analyzed.

2.7. Image Analysis. TEM image analysis was conducted in ImageJ (1.38 e/Java_1.5.0_09). The original pictures were processed with the "Subtract Background" and "Gaussian blur" filters to minimize noise. After adjusting the threshold, particles with high contrast remained, and the area of each particle was recorded using the "Analyze Particles" function. The particle area range was set to $0.0078-0.78 \mu \mathrm{m}^{2}$, which corresponds to the diameter range from $100 \mathrm{~nm}$ to $1 \mu \mathrm{m}$, assuming all particles are spherical.

The SEM "mapping" images were analyzed in ImageJ with manual labeling of all MPFs and fibrils. In total, nine "mapping" images were examined, where $100 \mathrm{MPFs}$ and 3347 fibrils were identified. From the abrasion test filters, 50 MPFs and 50 fibrils were sampled to assess their diameter. 


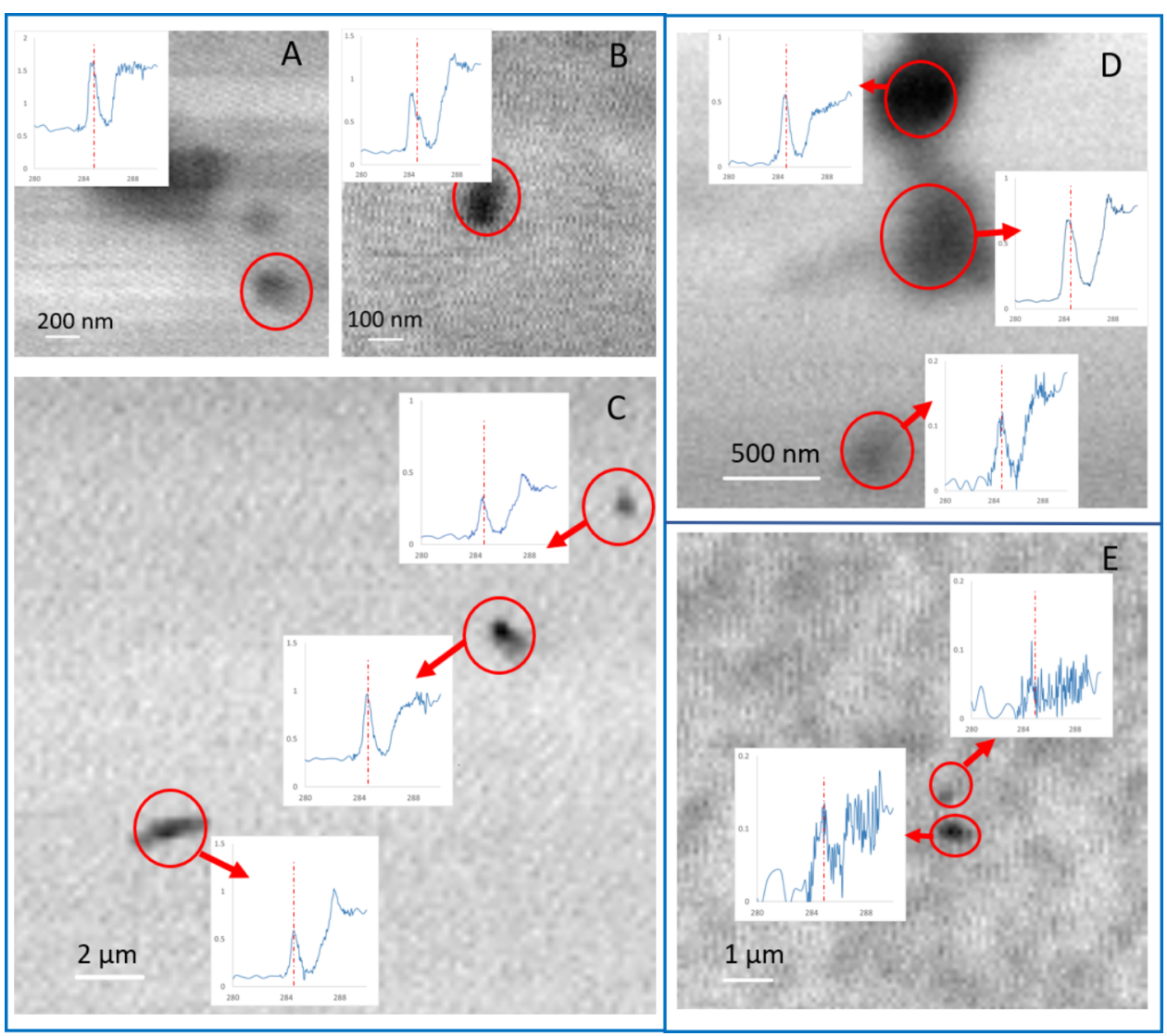

Figure 2. STXM image of nanoparticles on different sample grids with NEXAFS spectra next to the scanned particles: (A), (B), and (C) from abrasion samples; (D) from washing samples; and (E) from method blank sample.

Two fiber morphologies were identified. The first type had a cylindrical shape and is referred to as "MPF" in the text. The second type had a "tape-like" shape and a much smaller diameter. They are referred to as "fibrils". ${ }^{34}$

2.8. Statistics. The difference in nanoplastics number and mass released from different treatment groups was tested by the Welch two-sample $t$-test. The Welch two-sample $t$-test was also used to compare the difference of mean in two groups with a small sample size $(n<30)$. We conducted the analysis in $\mathrm{R}$ (version 4.0.5). A $p$-value of less than 0.05 was considered statistically significant. The normality of samples was tested in $\mathrm{R}$ by the Shapiro-Wilk method. A p-value of greater than 0.05 indicated that the sample distribution was not significantly different from a normal distribution. The Shapiro-Wilk test results can be found in Table S1.

\section{RESULTS}

3.1. Chemical Information. In total, we analyzed 30 STXM images of nanoparticles from our reference, washing, abrasion, and blank grids. The carbon edge is barely visible under low magnification; therefore, finding the particles was time-consuming, and scanning for NEXAFS took about $30 \mathrm{~min}$ per particle of interest. We obtained NEXAFS spectra of five PET reference nanoparticles with sizes ranging from 100 to $800 \mathrm{~nm}$. Peripheral areas of the particles were blurred, and thus it was impossible to report the exact size of measured nanoparticles. All those NEXAFS spectra have similar feature peaks. In Figure 1, we compare the NEXAFS spectrum of a selected PET nanoplastics ("line scan" in Figure 1A) with a calibrated PET NEXAFS spectrum with its characteristic features obtained from the literature. ${ }^{39}$ The most prominent feature of the spectrum is a $\pi^{*} \mathrm{C}=\mathrm{C}$ bond from the aromatic ring in the PET structure at an energy of 284.4 and $285.1 \mathrm{eV}$. The other peaks identified in the literature spectrum are $\pi^{*} \mathrm{C}=\mathrm{O}$ at $287.8 \mathrm{eV}$ and $\sigma^{*} \mathrm{C}-\mathrm{H}$ at $287.0 \mathrm{eV}$. The NEXAFS spectra of our PET reference particle match those peaks.

Twelve nanoparticles from three washing grids, 11 nanoparticles from three abrasion sample grids, and two nanoparticles from the method blank grid were identified on STXM images. All nanoparticles, except for two nanoparticles on the blank grid, were identified to be PET nanoplastics. Selected STXM images of nanoparticle clusters from abrasion, washing, and method blank grids are shown in Figure 2. Next to the identified particles, NEXAFS spectra (absorption signals from 280 to $290 \mathrm{eV}$ ) are plotted. The smallest particles that were identified to be PET were around $100 \mathrm{~nm}$ (smaller particles could not be analyzed by STXM). Unlike the NEXAFS spectra of the nanoparticles on the washing and abrasion sample grids, the aromatic ring feature peaks are missing in the spectra of the two nanoparticles from the blank sample grids (Figure 2E). However, the feature peaks in the rest of the NEXAFS spectra vary in relative intensity compared with that of the PET reference particles. The change in relative intensity of the feature peaks is likely explained by the influence of the thickness of the PET particle and the presence of a surfactant, LAS in our case, added during the washing process. Furthermore, in most of the spectra, there is only one peak at $285 \mathrm{eV}$ instead of two peaks at 284.4 and $285.1 \mathrm{eV}$. This is because the X-ray scanning is discrete, and, therefore, the signals were fitted into a smooth line with the contribution of 


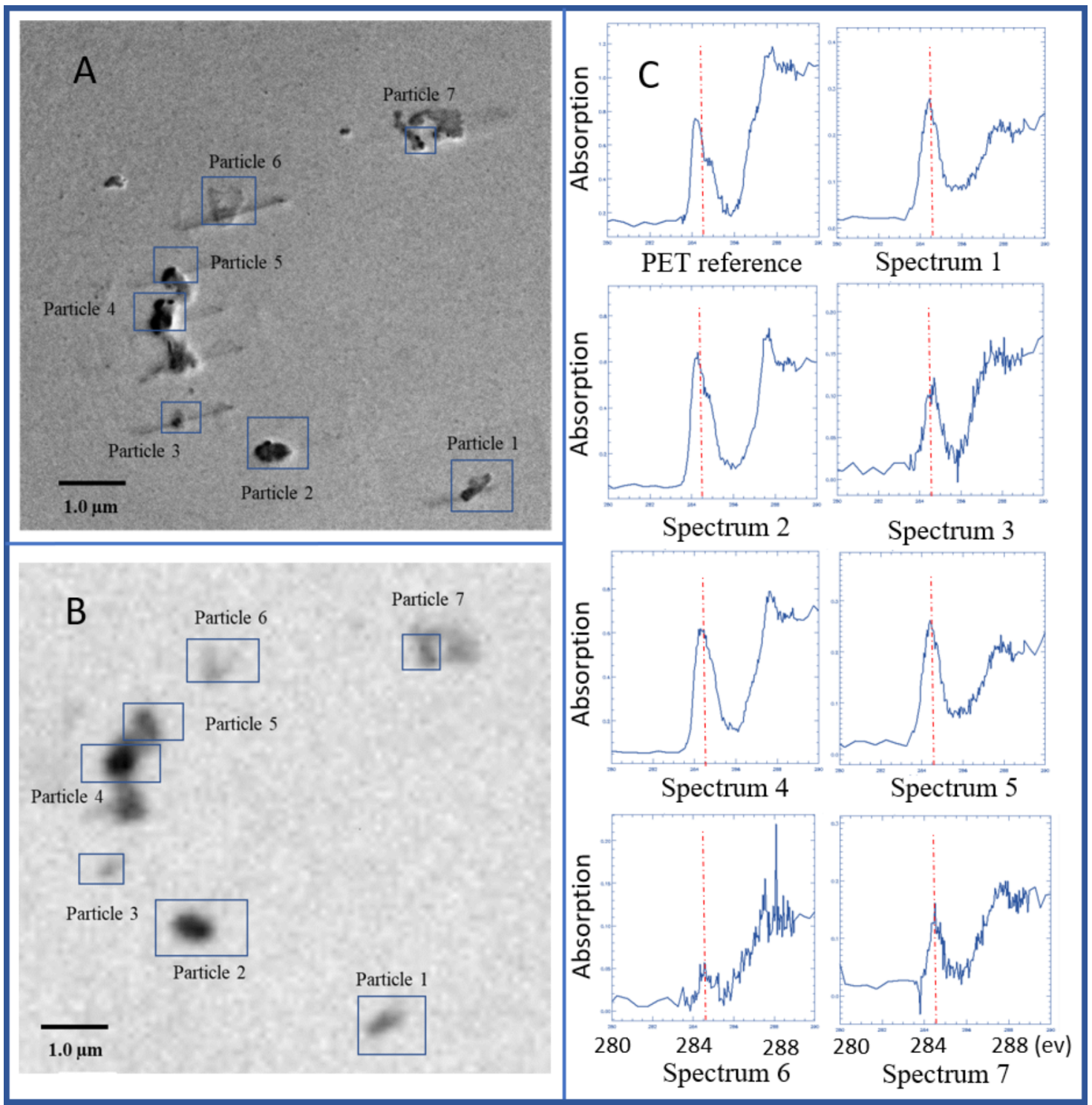

Figure 3. Comparison of (A) TEM and (B) STXM images of the same region of interest from a washing sample grid. Seven particles were identified and scanned for their corresponding NEXAFS spectra (C). For comparison, the NEXAFS spectrum of a PET reference NP is also shown.

the two peaks into one peak. ${ }^{39}$ To sum up, we can be confident of the existence of PET nanoplastics in both washing and abrasion samples.

3.2. Comparison of STXM and TEM Images. To better observe the morphologies of the PET nanoplastics and facilitate the physical characterization, the TEM and STXM images of the same region of interest is compared in Figure 3. For each of the seven particles, the corresponding NEXAFS spectra are shown. The bandwidth of the NEXAFS spectrum was cut from 280 to $290 \mathrm{eV}$ with a vertical line at $285 \mathrm{eV}$, indicating the PET feature peak. The TEM image has a higher resolution and sharper particle edges than the STXM image, enabling a more accurate size analysis. The lines across the particle in Figure $3 \mathrm{~A}$ are the visible beam damage from the NEXAFS line scans (the TEM image was taken after the STXM analysis). The NEXAFS spectra of particles 1, 2, 4, and 5 are similar to the spectrum of the PET reference. These particles also have a higher contrast in both the TEM and STXM images, indicative of a larger size. On the contrary, particles 3 and 7 show less contrast as well as a lower intensity of the feature peak at around $285 \mathrm{eV}$. Moreover, particle 6 exhibits almost no carbon signal, resulting in a low peak at 285 $\mathrm{eV}$. These particles are likely flakes and therefore have only a small thickness, resulting in low contrast. Figure 3, therefore, provides us with important morphological information of the PET nanoplastics released during washing and abrasion of textiles: the released nanoplastics are of irregular shape, but many are nearly spherical. We cannot confirm that all of the nanoparticles in our samples are PET nanoplastics; however, as visible in Figure 3, the majority of the nanoparticles (6 out of 7 particles in this case) have been confirmed to be actually PET nanoplastics.

3.3. Morphology and Size. The morphology of nanoplastics was characterized by TEM. Selected TEM images at $4000 \times$ and $40000 \times$ magnification can be found in Figure S5. There are apparent agglomerates of PET nanoplastics in both washing and abrasion samples, as shown in Figure S5A,C. When zooming into $40000 \times$ magnification (Figure S5B), the agglomerates are more distinguishable. However, many nanoplastics are potentially single particles with a size smaller 


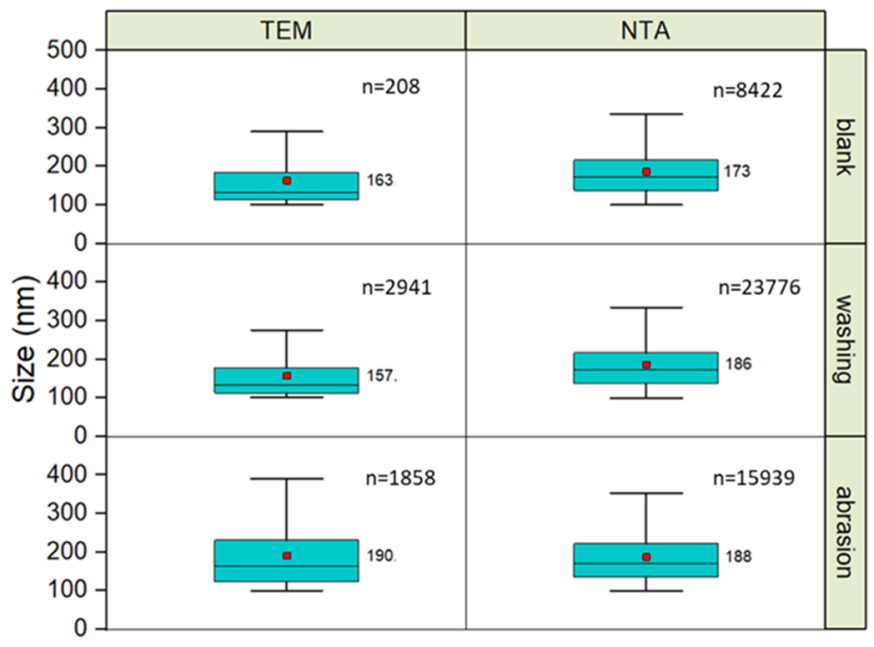

B

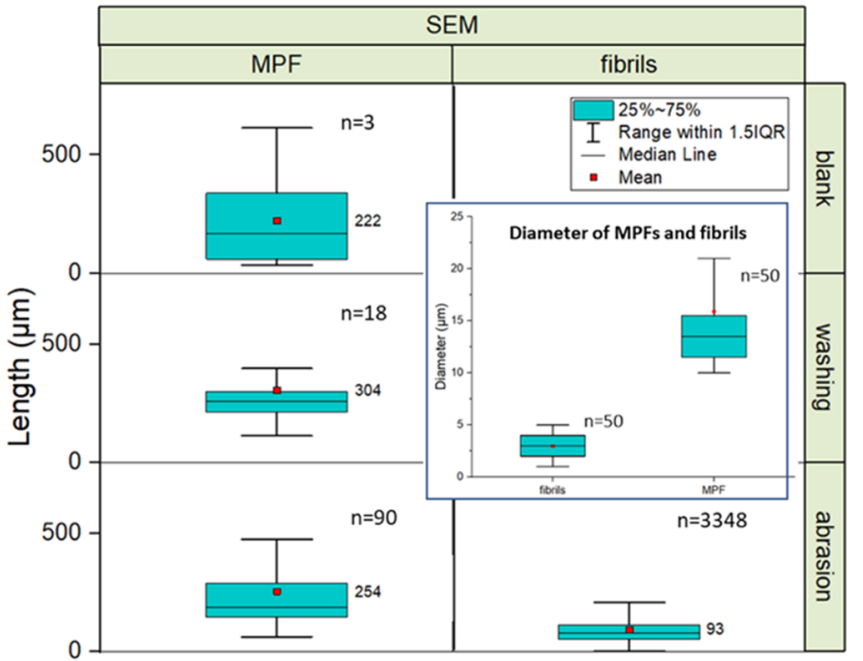

Figure 4. Length and size boxplot of the released nanoparticles (A) and MPFs/fibrils (B). The boxplot covers $25-75 \%$ of the size/length with median line and mean fiber length $(\mu \mathrm{m}) / \mathrm{NP}$ size $(\mathrm{nm})$ value labeled. The number of particles used for each boxplot is given as " $n=$ the sample size." There are no fibrils identified in the blank and washing samples.

Table 1. Number of Nanoplastics/MPFs/Fibrils Released Per Gram of Textiles and the Mass (mg) of Nanoplastics/MPFs/ Fibrils Released Per Gram of Textiles from Washing and Abrasion Samples ${ }^{a}$

\begin{tabular}{|c|c|c|c|c|c|c|}
\hline $\begin{array}{l}\text { characterization } \\
\text { method }\end{array}$ & plastic type & sample & $\begin{array}{l}\text { number concentration } \\
\text { (number/g textile) }\end{array}$ & $\begin{array}{l}\text { standard deviation } \\
\text { (number/g textile) }\end{array}$ & $\begin{array}{l}\text { mass concentration } \\
(\mathrm{mg} / \mathrm{g} \text { textile })\end{array}$ & $\begin{array}{l}\text { standard deviation } \\
\text { (mg/g textile) }\end{array}$ \\
\hline \multirow[t]{2}{*}{ NTA } & \multirow[t]{2}{*}{ nanoplastics } & washing & $3.3 \times 10^{11}$ & $6 \times 10^{10}$ & 2.1 & 0.3 \\
\hline & & abrasion & $2.1 \times 10^{11}$ & $3 \times 10^{10}$ & 1.4 & 0.6 \\
\hline \multirow[t]{3}{*}{ SEM } & \multirow[t]{2}{*}{ MPFs } & washing & 2860 & 670 & 0.2 & 0.05 \\
\hline & & abrasion & 14100 & 1500 & 1.0 & 0.1 \\
\hline & fibrils & abrasion & $5.3 \times 10^{5}$ & $4.8 \times 10^{4}$ & 0.44 & 0.04 \\
\hline
\end{tabular}

${ }^{a_{T}}$ The conversion from number to mass was based on the assumption that nanoplastics are spherical and that MPFs/fibrils are cylindrical. The particle concentrations in the blank samples were subtracted from all samples.

than $100 \mathrm{~nm}$, as can been seen in Figure S5C,D. The released nanoplastics are irregular but almost spherical, and the more contrast a nanoparticle has, the more likely it is a nanoplastic, according to the comparison between the STXM and TEM image shown in Figure 3. There are also particles in Figure 3A, to the left of particle 6 and one to the left of particle 7, that are captured by the TEM image while being barely visible in the STXM image. Those particles are likely noncarbon contaminants that are counted by the TEM image analysis and by NTA in the method blank. Based on this feature, we analyzed the TEM images by ImageJ by manipulating the contrast (as demonstrated in Figure S3) to characterize the size of nanoparticles. A total number of 135 TEM images were analyzed using ImageJ to obtain the size information. About 2500 particles were recorded, ranging in size mostly from 100 to $500 \mathrm{~nm}$. We excluded in the image analysis process nanoparticles smaller than $100 \mathrm{~nm}$ because the minimal size of PET nanoplastics we could clearly identify in the STXM images to be composed of PET was around $100 \mathrm{~nm}$. The average size of the nanoparticles released during washing and abrasion was determined to be $158 \pm 63$ and $190 \pm 90 \mathrm{~nm}$, respectively, while in the blank samples, the average size of the nanoparticles was $163 \pm 81 \mathrm{~nm}$.

We also quantified the size of the nanoparticles using NTA, and the size distribution results were converted into boxplots (Figure 4A). The size distribution raw data from the NTA can be found in Figure S6, with the contribution from the blank samples subtracted. The mean size of the nanoparticles in washing and abrasion samples, reported by NTA, was $186 \pm$ 65 and $188 \pm 68 \mathrm{~nm}$, respectively (Figure $4 \mathrm{~A}$ ). The peaks of the size distribution for the washing and abrasion samples are similar, both at around $165 \mathrm{~nm}$ (Figure S6).

Under SEM (Figure S7), the morphologies of MPFs and fibrils are easily distinguishable. MPFs are thick cylinders, while the fibrils are slim and long "tapes." MPFs have an average diameter of $16 \pm 7 \mu \mathrm{m}$, while fibrils have an average diameter of $3 \pm 1 \mu \mathrm{m}$. We only found fibrils in the abrasion samples, and their average length of $93 \pm 70 \mu \mathrm{m}$ is much smaller than the length of the MPFs, $254 \pm 200 \mu \mathrm{m}$. Washing samples released MPFs with a length of $304 \pm 270 \mu \mathrm{m}$. There are only very few MPFs $(n=3)$, and no fibrils are identified in the blank samples.

3.4. Number and Mass Concentration of Released Particles. The number of nanoparticles released during washing and abrasion of polyester textiles was determined by NTA. The instrument software reports the concentration-size distribution from 1 to $2000 \mathrm{~nm}$ fitted with a smooth curve. We summed up the concentrations from 100 to $1000 \mathrm{~nm}$ reported by NTA to get the total number of nanoparticles released from method blank, washing samples, and abrasion samples. In Table 1, we compare the number of released MPFs and fibrils obtained from SEM image analysis with the number of released nanoplastics. Typically, $2.1 \pm 0.3 \times 10^{11}$ and $3.3 \pm 0.6 \times 10^{11}$ nanoplastics/g textile were released from abrasion and washing 
samples, respectively. However, there was a substantial release of $2.8 \pm 0.6 \times 10^{8}$ nanoparticles $/ \mathrm{mL}$ from the control group (for example, noncarbon nanoparticles captured in Figure 3A). This blank contribution was subtracted from all samples. We were not able to eliminate this part of contamination, but the increase in nanoparticle concentration compared to the blank sample by washing $(p=0.006)$ and abrasion $(p=0.03)$ was statistically significant. The number of MPFs extracted from the fleece polyester textiles in washing and abrasion samples was $2860 \pm 670$ and $14100 \pm 1500 \mathrm{MPFs} / \mathrm{g}$ textile, respectively. In abrasion samples, a concentration of fibrils of $5.31 \pm 0.48 \times 10^{5} / \mathrm{g}$ textiles was identified, which is about $30-$ 40 times the number of MPFs that are released. There were no fibrils found in neither the blank nor the washing samples, indicating that only abrasion contributes to the release of fibrils.

The mass of nanoplastics/MPFs/fibrils released was calculated and the values are presented in Table 1 . The mass calculation of nanoplastics was based on the size distribution (bin interval at $1 \mathrm{~nm}$ ) reported by NTA (Figure S6) and on the assumption that the nanoplastics are spherical and the $\mathrm{MPF} /$ fibrils are cylindrical. We estimated that $1 \mathrm{~g}$ of polyester fleece textile released about $1.4 \pm 0.6 \mathrm{mg}$ of nanoplastics (with abrasion) and $2.1 \pm 0.3 \mathrm{mg}$ of nanoplastics (without abrasion) during washing. There was no significant difference $(p=0.4)$ in the mass of the nanoplastics released between washing and abrasion samples. Meanwhile, there was a significant increase $(p=0.003)$ of MPFs released after abrasion. The mass of MPFs released from washing and abrasion samples were 0.20 \pm 0.05 and $1.0 \pm 0.1 \mathrm{mg}$, respectively. Furthermore, the release of fibrils was only detected in abrasion samples with $0.44 \pm$ $0.04 \mathrm{mg} / \mathrm{g}$ textile. From all reported mass release values, the blank values were subtracted, and, therefore, the net contribution of abrasion and washing is reported.

\section{DISCUSSION}

The present study is a continuation of previous microplastic release studies from polyester textiles during washing and abrasion, ${ }^{32,34}$ with an adoption of the method of washing and abrasion to also characterize the smaller-sized fraction. We used different techniques to characterize the released nanoparticles, both physically and chemically. The present study is the first to identify PET nanoplastics released during abrasion and washing of polyester textiles.

The comparison of the STXM and NEXAFS spectra between the reference PET nanoplastics and the nanoparticles released from the textiles confirmed the presence of PET nanoplastics in the washing solution of polyester textiles with or without abrasion. Different to studies characterizing nanoplastics in environmental samples, we have a defined system using $100 \%$ polyester textiles as starting materials. We, therefore, used the STXM analysis to confirm the presence of PET nanoplastics and exclude contamination by other particles rather than having to identify polymers in an unknown sample. STXM has been used before to identify the chemical identity of individual nanoplastic particles: Bigalke et al. reported the presence of polystyrene nanoplastic fibers in soil samples using this method. ${ }^{40}$ The advantage of STXM lies in the chemical identification at the single-particle level. The NEXAFS spectra of nanoparticles obtained from textiles samples were all representative for PET, while those for particles from the control samples were not. The minimal size of identifiable PET nanoplastics by STXM is around $100 \mathrm{~nm}$ due to the limited resolution. In the present study, we therefore quantified only particles above $100 \mathrm{~nm}$ to make sure that what we counted are indeed PET nanoplastics, and we refrain from making any statements about the smaller size fraction.

Our TEM images captured both agglomerated PET nanoplastics and single-particle PET nanoplastics. The hydrodynamic size of the nanoparticles released from textiles in the present study ranged from 150 to $190 \mathrm{~nm}$ and was similar to the size of $180 \mathrm{~nm}$ for surfactant-capped PET nanoplastics generated by mechanical grinding of PET bottles reported by Ji et al. ${ }^{41} \mathrm{Li}$ et al. found that the nanoplastics released from polypropylene milk bottles during the preparation of powdered milk had a mean particle diameter of $\sim 100 \mathrm{~nm} .{ }^{15}$ Lambert et al. also found, by NTA, that most of the polystyrene nanoplastics formed during the UV degradation of a coffee cup lid were smaller than $500 \mathrm{~nm}$, with an average size of 224 nm. ${ }^{11}$

With regard to the quantification of microplastics and nanoplastics, we estimated that in the abrasion samples, $1 \mathrm{~g}$ of textile released an average of $2.1 \times 10^{11}$ nanoplastics, $1.4 \times 10^{4}$ MPFs, and $5.3 \times 10^{5}$ fibrils. In the washing samples, $1 \mathrm{~g}$ of textile released an average of $3.3 \times 10^{11}$ nanoplastics, $2.8 \times 10^{3}$ MPFs, and no fibrils. The number of fibrils was about 38 times the number of MPFs, similar to the ratio of about 30 reported by Cai et al. ${ }^{34}$ Several studies reported numbers of nanoplastics released from different products. It was estimated that one plastic teabag at brewing temperature $\left(95{ }^{\circ} \mathrm{C}\right)$ released 2.3 million microplastic particles $(>1 \mu \mathrm{m})$ and 14.7 billion nanoplastic particles $(<1 \mu \mathrm{m}$ in size $),{ }^{14}$ which is equivalent to $2.3 \times 10^{7}$ microplastics/g teabag (provided the average weight of an empty teabag is $0.1 \mathrm{~g}$ ) and $1.5 \times 10^{10}$ nanoplastics/g teabag. The present study showed that the number of nanoplastics (per $\mathrm{g}$ of textile) released during washing and abrasion of polyester textiles was about 1 order of magnitude higher than that from the teabags, while the number of microplastics was 3-4 orders of magnitude lower. The number concentration of nanoplastics released from polypropylene milk bottles during the preparation of powder milk ${ }^{15}$ was estimated to be $2.5 \times 10^{10}$ particles/g milk bottle (assuming $250 \mathrm{~mL}$ liquid and $10 \mathrm{~g}$ bottle weight), which is also 1 order of magnitude lower than the number concentration released from textiles in the present study. After 56 days of UV degradation, a coffee cup lid was found to release $8 \times 10^{10}$ particles/g coffee lid (calculated taking $20 \mathrm{~mL}$ water and $1 \mathrm{~cm}^{2}$ lid based on the description given in the study), ${ }^{11}$ which is $25-$ $40 \%$ of the number of nanoplastics released per gram of textiles found in the present study. The number of nanoplastic particles released from textiles is, therefore, a bit larger than those from other plastic types or articles. A main difference between the previous studies and ours is that textile is composed of yarns that are spun from single staple fibers and therefore has a much larger surface area than a milk bottle or a coffee lid, which both have a smooth surface. This difference in structure may explain the higher release when normalized to the weight, as the surface area is not available for all of the studied materials.

The weight of MPFs released (with or without abrasion) from the polyester fleece textiles was in the same order of magnitude $(0.1-1 \mathrm{mg} / \mathrm{g}$ textile) as in many other published washing studies. $^{26,30,32,33}$ In total, 2.9 and $2.3 \mathrm{mg}$ micro- and nanoplastics were released from textiles (with or without abrasion) during washing of $1 \mathrm{~g}$ of polyester fleece textile. Nanoplastics accounted for nearly $50 \%$ of the weight in the 
abrasion samples and more than $90 \%$ weight in the washing samples. The present study is the first to report the amount of nanoplastic released during the use of products on a weight basis and shows that nanoplastics can be a considerable weight fraction of the released plastic particles.

The studies mentioned above, including the present study, indicated that a high concentration of nanoplastics can be released during the daily use of plastic products. The World Health Organization published a review on the plastic contamination of drinking water, which reported an estimated concentration of a few tens to a few hundred microplastic particles per liter. ${ }^{42}$ The nanoplastic particles are orders of magnitude larger based on particle numbers and can reach the same order of magnitude in terms of mass. Up to $99 \%$ of the micro- and nanoplastics can be removed in the wastewater treatment processes according to a study, which used metaldoped nanoplastics to simulate the fate of nanoplastics in wastewater treatment plants. ${ }^{43}$ Yet even a small percentage of nanoplastics that is not retained and which will eventually make it into the environment may still be relevant in terms of particle number. Although a meta-analysis of published ecotoxicity studies for nanoplastics has found that their predicted no-effect concentration (PNEC) is higher than the one of microplastics, ${ }^{22}$ the environmental risk of nanoplastics cannot be ignored due to potentially much higher particle number concentrations in the aqueous environment.

There are some limitations of the present study. From the NTA results, we know that there are only very few particles between $400 \mathrm{~nm}$ and $2 \mu \mathrm{m}$. The fibrils that were quantified have diameters of $3 \pm 1 \mu \mathrm{m}$. What is missing is, therefore, microplastic particles (nonfibrous debris) with a size larger than $2 \mu \mathrm{m}$ to configure a full profile of the plastic emissions from textiles during washing and abrasion. So far, we also only investigated the release from one type of fleece fabric in the present study. Fleece fabrics were found to release much more $\mathrm{MPFs}^{32}$ than most other fabric types and may therefore also release more nanoplastics. A systematic study should be done to study more factors influencing the release of nanoplastics from textiles, including the fabric structure, number of wash cycles, mechanical stress, and the volume of the wash solution. Last but not least, it is counter-intuitive to see that there was no difference in the number of nanoplastics released from textiles before and after abrasion. The abrasion process was producing fibrils, so it resulted in the partial destruction of the fibers. However, this does not seem to influence the number of nanoplastics released from the fleece fabric. Future studies with various fabric structures and under different conditions need to establish the process by which the nanoplastics are formed and released from textiles. It has been established that the MPFs that are released during washing or after abrasion are actually not formed during the washing or abrasion process but were already present in the textile and were just liberated. ${ }^{32,44} \mathrm{~A}$ study using an extraction protocol was able to show that the MPFs which could be released from textiles were already contained in the finished fabric and were also found in the fibers and yarns used to produce the textiles. ${ }^{44}$ It is, therefore, possible that the nanoplastics released during washing also originate from the production process, which could explain why the abrasion process has no influence on their amount.

\section{ASSOCIATED CONTENT}

\section{Supporting Information}

The Supporting Information is available free of charge at https://pubs.acs.org/doi/10.1021/acs.est.1c04826.

Details about experimental methods; numerical data on the number, size, and mass of particles; and additional TEM and SEM images (PDF)

\section{AUTHOR INFORMATION}

\section{Corresponding Author}

Bernd Nowack - Technology and Society Laboratory, Empa - Swiss Federal Laboratories for Materials Science and Technology, 9014 St. Gallen, Switzerland; (1) orcid.org/ 0000-0002-5676-112X; Phone: +41 58765 7692;

Email: nowack@empa.ch

\section{Authors}

Tong Yang - Technology and Society Laboratory, Empa Swiss Federal Laboratories for Materials Science and Technology, 9014 St. Gallen, Switzerland

Jialuo Luo - Laboratory for Biomimetic Membranes and Textiles, Empa - Swiss Federal Laboratories for Materials Science and Technology, 9014 St. Gallen, Switzerland

Complete contact information is available at:

https://pubs.acs.org/10.1021/acs.est.1c04826

\section{Notes}

The authors declare no competing financial interest.

\section{ACKNOWLEDGMENTS}

The authors would like to thank Brian Sinnet from Eawag for the facilitation of TEM grid preparation. Funding for beamtime at the PolLux beamline at the Swiss Light Source (SLS) in Villigen, Switzerland, was provided through proposal ID 20200475. The help of Barbora Pinlova during the visit to SLS is greatly acknowledged.

\section{REFERENCES}

(1) Ivar do Sul, J. A.; Labrenz, M. Microplastics into the Anthropocene. In Handbook of Microplastics in the Environment; Rocha-Santos, T.; Costa, M.; Mouneyrac, C., Eds.; Springer International Publishing: Cham, 2020; pp 1-16.

(2) Thompson, R. C.; Olsen, Y.; Mitchell, R. P.; Davis, A.; Rowland, S. J.; John, A. W. G.; McGonigle, D.; Russell, A. E. Lost at Sea: Where Is All the Plastic? Science 2004, 304, 838.

(3) Kershaw, P. Sources, Fate and Effects of Microplastics in the Marine Environment: A Global Assessment; International Maritime Organization, 2015; pp 1020-4873.

(4) Hurley, R.; Woodward, J.; Rothwell, J. J. Microplastic contamination of river beds significantly reduced by catchment-wide flooding. Nat. Geosci. 2018, 11, 251-257.

(5) Koelmans, A. A.; Besseling, E.; Shim, W. J. Nanoplastics in the Aquatic Environment. Critical Review. In Marine Anthropogenic Litter; Bergmann, M.; Gutow, L.; Klages, M., Eds.; Springer International Publishing: Cham, 2015; pp 325-340.

(6) Hernandez, L. M.; Yousefi, N.; Tufenkji, N. Are There Nanoplastics in Your Personal Care Products? Environ. Sci. Technol. Lett. 2017, 4, 280-285.

(7) Hartmann, N. B.; Hüffer, T.; Thompson, R. C.; Hassellöv, M.; Verschoor, A.; Daugaard, A. E.; Rist, S.; Karlsson, T.; Brennholt, N.; Cole, M.; Herrling, M. P.; Hess, M. C.; Ivleva, N. P.; Lusher, A. L.; Wagner, M. Are We Speaking the Same Language? Recommendations for a Definition and Categorization Framework for Plastic Debris. Environ. Sci. Technol. 2019, 53, 1039-1047. 
(8) Frias, J. P. G. L.; Nash, R. Microplastics: Finding a consensus on the definition. Mar. Pollut. Bull. 2019, 138, 145-147.

(9) Mitrano, D. M.; Wick, P.; Nowack, B. Placing nanoplastics in the context of global plastic pollution. Nat. Nanotechnol. 2021, 16, 491500.

(10) Gigault, J.; El Hadri, H.; Nguyen, B.; Grassl, B.; Rowenczyk, L.; Tufenkji, N.; Feng, S.; Wiesner, M. Nanoplastics are neither microplastics nor engineered nanoparticles. Nat. Nanotechnol. 2021, 16, 501-507.

(11) Lambert, S.; Wagner, M. Characterisation of nanoplastics during the degradation of polystyrene. Chemosphere 2016, 145, 265268.

(12) Ekvall, M. T.; Lundqvist, M.; Kelpsiene, E.; Šileikis, E.; Gunnarsson, S. B.; Cedervall, T. Nanoplastics formed during the mechanical breakdown of daily-use polystyrene products. Nanoscale Adv. 2019, 1, 1055-1061.

(13) Dawson, A. L.; Kawaguchi, S.; King, C. K.; Townsend, K. A.; King, R.; Huston, W. M.; Bengtson Nash, S. M. Turning microplastics into nanoplastics through digestive fragmentation by Antarctic krill. Nat. Commun. 2018, 9, No. 1001.

(14) Hernandez, L. M.; Xu, E. G.; Larsson, H. C. E.; Tahara, R.; Maisuria, V. B.; Tufenkji, N. Plastic Teabags Release Billions of Microparticles and Nanoparticles into Tea. Environ. Sci. Technol. 2019, 53, 12300-12310.

(15) Li, D.; Shi, Y.; Yang, L.; Xiao, L.; Kehoe, D. K.; Gun'ko, Y. K.; Boland, J. J.; Wang, J. J. Microplastic release from the degradation of polypropylene feeding bottles during infant formula preparation. Nat. Food 2020, 1, 746-754.

(16) Cai, H.; Xu, E. G.; Du, F.; Li, R.; Liu, J.; Shi, H. Analysis of environmental nanoplastics: Progress and challenges. Chem. Eng. J. 2021, 410, No. 128208.

(17) Wahl, A.; Le Juge, C.; Davranche, M.; El Hadri, H.; Grassl, B.; Reynaud, S.; Gigault, J. Nanoplastic occurrence in a soil amended with plastic debris. Chemosphere 2021, 262, No. 127784.

(18) Ter Halle, A.; Jeanneau, L.; Martignac, M.; Jardé, E.; Pedrono, B.; Brach, L.; Gigault, J. Nanoplastic in the North Atlantic Subtropical Gyre. Environ. Sci. Technol. 2017, 51, 13689-13697.

(19) Materić, D.; Kasper-Giebl, A.; Kau, D.; Anten, M.; Greilinger, M.; Ludewig, E.; van Sebille, E.; Röckmann, T.; Holzinger, R. Microand Nanoplastics in Alpine Snow: A New Method for Chemical Identification and (Semi)Quantification in the Nanogram Range. Environ. Sci. Technol. 2020, 54, 2353-2359.

(20) Sun, X.-D.; Yuan, X.-Z.; Jia, Y.; Feng, L.-J.; Zhu, F.-P.; Dong, S.S.; Liu, J.; Kong, X.; Tian, H.; Duan, J.-L.; Ding, Z.; Wang, S.-G.; Xing, B. Differentially charged nanoplastics demonstrate distinct accumulation in Arabidopsis thaliana. Nat. Nanotechnol. 2020, 15, $755-760$.

(21) Cui, R.; Kim, S. W.; An, Y.-J. Polystyrene nanoplastics inhibit reproduction and induce abnormal embryonic development in the freshwater crustacean Daphnia galeata. Sci. Rep. 2017, 7, No. 12095.

(22) Yang, T.; Nowack, B. A Meta-analysis of Ecotoxicological Hazard Data for Nanoplastics in Marine and Freshwater Systems. Environ. Toxicol. Chem. 2020, 39, 2588-2598.

(23) Koelmans, A. A.; Mohamed Nor, N. H.; Hermsen, E.; Kooi, M.; Mintenig, S. M.; De France, J. Microplastics in freshwaters and drinking water: Critical review and assessment of data quality. Water Res. 2019, 155, 410-422.

(24) Coyle, R.; Hardiman, G.; Driscoll, K. O. Microplastics in the marine environment: A review of their sources, distribution processes, uptake and exchange in ecosystems. Case Stud. Chem. Environ. Eng. 2020, 2, No. 100010.

(25) Kawecki, D.; Nowack, B. Polymer-Specific Modeling of the Environmental Emissions of Seven Commodity Plastics As Macroand Microplastics. Environ. Sci. Technol. 2019, 53, 9664-9676.

(26) Hernandez, E.; Nowack, B.; Mitrano, D. M. Polyester Textiles as a Source of Microplastics from Households: A Mechanistic Study to Understand Microfiber Release During Washing. Environ. Sci. Technol. 2017, 51, 7036-7046.
(27) Carney Almroth, B. M.; Åström, L.; Roslund, S.; Petersson, H.; Johansson, M.; Persson, N.-K. Quantifying shedding of synthetic fibers from textiles; a source of microplastics released into the environment. Environ. Sci. Pollut. Res. 2018, 25, 1191-1199.

(28) De Falco, F.; Gullo, M. P.; Gentile, G.; Di Pace, E.; Cocca, M.; Gelabert, L.; Brouta-Agnésa, M.; Rovira, A.; Escudero, R.; Villalba, R.; Mossotti, R.; Montarsolo, A.; Gavignano, S.; Tonin, C.; Avella, M. Evaluation of microplastic release caused by textile washing processes of synthetic fabrics. Environ. Pollut. 2018, 236, 916-925.

(29) De Falco, F.; Di Pace, E.; Cocca, M.; Avella, M. The contribution of washing processes of synthetic clothes to microplastic pollution. Sci. Rep. 2019, 9, No. 6633.

(30) Kelly, M. R.; Lant, N. J.; Kurr, M.; Burgess, J. G. Importance of Water-Volume on the Release of Microplastic Fibers from Laundry. Environ. Sci. Technol. 2019, 53, 11735-11744.

(31) McIlwraith, H. K.; Lin, J.; Erdle, L. M.; Mallos, N.; Diamond, M. L.; Rochman, C. M. Capturing microfibers - marketed technologies reduce microfiber emissions from washing machines. Mar. Pollut. Bull. 2019, 139, 40-45.

(32) Cai, Y.; Yang, T.; Mitrano, D. M.; Heuberger, M.; Hufenus, R.; Nowack, B. Systematic Study of Microplastic Fiber Release from 12 Different Polyester Textiles during Washing. Environ. Sci. Technol. 2020, 54, 4847-4855.

(33) Kärkkäinen, N.; Sillanpää, M. Quantification of different microplastic fibres discharged from textiles in machine wash and tumble drying. Environ. Sci. Pollut. Res. 2021, 28, 16253-16263.

(34) Cai, Y.; Mitrano, D. M.; Hufenus, R.; Nowack, B. Formation of Fiber Fragments during Abrasion of Polyester Textiles. Environ. Sci. Technol. 2021, 55, 8001-8009.

(35) Rodríguez-Hernández, A. G.; Munoz-Tabares, J. A.; AguilarGuzman, J. C.; Vazquez-Duhalt, R. A novel and simple method for polyethylene terephthalate (PET) nanoparticle production. Environ. Sci.: Nano 2019, 6, 2031-2036.

(36) ISO, Standard 12947-2:2016. Textiles - Determination of the Abrasion Resistance of Fabrics by the Martindale Method - Part 2: Determination of Specimen Breakdown, 2016.

(37) Textor, T.; Derksen, L.; Bahners, T.; Gutmann, J. S.; MayerGall, T. Abrasion resistance of textiles: Gaining insight into the damaging mechanisms of different test procedures J. Eng. Fibers Fabrics 2019 14, 1558925019829481, DOI: 10.1177/ 1558925019829481.

(38) ISO, 105-C06: Textiles - Tests for Colour Fastness Part C06: Colour Fastness to Domestic and Commercial Laundering, 1994.

(39) Dhez, O.; Ade, H.; Urquhart, S. G. Calibrated NEXAFS spectra of some common polymers. J. Electron Spectrosc. Relat. Phenom. 2003, $128,85-96$.

(40) Bigalke, M.; Filella, M.; Fischer, D.; Muntwyler, A.; Scheurer, M.; Watts, B. Micro- and Nanoplastic Analysis in Soils. Chimia 2018, $72,901$.

(41) Ji, Y.; Wang, C.; Wang, Y.; Fu, L.; Man, M.; Chen, L. Realistic polyethylene terephthalate nanoplastics and the size- and surface coating-dependent toxicological impacts on zebrafish embryos. Environ. Sci.: Nano 2020, 7, 2313-2324.

(42) Mason, S. A.; Welch, V. G.; Neratko, J. Synthetic Polymer Contamination in Bottled Water. Front. Chem. 2018, 6, No. 407.

(43) Frehland, S.; Kaegi, R.; Hufenus, R.; Mitrano, D. M. Long-term assessment of nanoplastic particle and microplastic fiber flux through a pilot wastewater treatment plant using metal-doped plastics. Water Res. 2020, 182, No. 115860 .

(44) Cai, Y.; Mitrano, D. M.; Heuberger, M.; Hufenus, R.; Nowack, B. The origin of microplastic fiber in polyester textiles: The textile production process matters. J. Cleaner Prod. 2020, 267, No. 121970. 